\title{
Mundwasser hilft gegen Tripper
}

\author{
Tägliche Mundspülungen mit handelsüblichem Mundwasser könnten einen Beitrag zur \\ Eindämmung der endemischen Verbreitung der Gonorrhoe bei MSM leisten.
}

\begin{abstract}
Die gute Behandelbarkeit der HIVInfektion hat die diesbezügliche Vorsicht erodiert und zu einem Rückgang des Gebrauchs von Kondomen geführt. Als Resultat belegen die Statistiken des Robert-Koch-Instituts, dass die Prävalenzen anderer sexuell übertragbarer Erkrankungen wie Gonorrhoe und Lues zunehmen. Motor der Entwicklung sind nach wie vor Männer, die Sex mit Männern haben (MSM).

Eine Studie aus Australien zeigt nun eine einfach anwendbare prophylaktische Maßnahme gegen die Verbreitung der Gonorrhoe auf. In einem In-vitroVersuch konnte zunächst die antimikrobielle Wirkung von Mundwasser des Herstellers Listerine ${ }^{\circledast}$ klar demonstriert werden. Somit konnte der bereits 1879 von der Firma behauptete, aber nie bewiesene Effekt nachgewiesen werden.
\end{abstract}

Auf der Basis dieser Ergebnisse führte man eine randomisierte, kontrollierte Studie mit 196 MSM durch, bei denen bereits früher einmal Neisseria gonorrhoeae in einem Rachenabstrich nachgewiesen worden war und die sich zur Therapie in einer Ambulanz für Geschlechtskrankheiten vorstellten.

58 Männern zeigten in der PCR einen immer noch positiven Rachen- und Tonsillenabstrich. 33 von ihnen spülten eine Minute lang mit Mundwasser, 25 mit Kochsalzlösung. Bei einem erneuten Abstrich fünf Minuten nach dieser Maßnahme war der Anteil der nach wie vor positiven Männer in der Verumgruppe auf 52\% zurückgegangen, in der Kochsalzgruppe dagegen nur auf $84 \%$.

- Chow EP et al. Antiseptic mouthwash against pharyngeal Neisseria gonorrhoeae: a randomised controlled trial and an in vitro study. Sex Transm Infect. 2017;93:88-93.

\section{KOMMENTAR}

Trotz zahlreicher methodischer Probleme - wie lange hält dieser Effekt eigentlich vor? - hat die Studie doch einen höchst interessanten Aspekt. Die Maßnahme der täglichen Anwendung von Mundwasser ist sehr leicht durchführbar und besitzt in dieser Risikogruppe sicher wesentlich höhere Akzeptanz als die Verwendung von Kondomen. Der Effekt mag gering sein, doch handelt es sich möglicherweise um eine vor allem epidemiologisch interessante Maßnahme. Männer mit pharyngealer Gonorrhoe suchen nämlich im Gegensatz zu Männern mit Urethritis viel seltener den Arzt auf, da sie kaum Beschwerden haben. Insofern spielen sie als Überträger wahrscheinlich eine größere Rolle als Patienten mit Urethritis.

Prof. Dr. med. H. S. FüeßI

\section{Sturz und akromioklavikulare Sprengung}

Ein 30-jähriger Mann suchte die Nothilfe auf, weil er bei einem Sturz auf die linke Schulter gefallen war. Bei der Untersuchung fand sich eine deutlich sichtbare Schwellung über der linken Schulter (Abb. A, B). Die aktive Abduktion der Schulter war schmerzhaft eingeschränkt. Am linken Arm lagen keine Hinweise für eine Durchblutungsstörung oder neurologische Ausfälle vor. Das Sternokla- vikulargelenk war nicht druckschmerzhaft. Auf einer p.a.-Aufnahme der linken Schulter erkannte man eine komplette Sprengung des Akromioklavikulargelenks (Abb. C).

Dieser Verletzungstyp wird in der Rockwood-Klassifikation als Typ IV bezeichnet. Ihm liegt eine Ruptur der Gelenkkapsel und des Ligamentum acromioclaviculare und coracoclaviculare zugrunde, wobei der distale Anteil der Clavicula durch den M. trapezius nach posterior disloziert wird. Das Gelenk wurde geschlossen reponiert und fixiert. Sechs Monate später konnte der Patient die Schulter wieder schmerzfrei voll bewegen.

Prof. Dr. med. H. S. FüeßI

- Kayaalp ME, Seker A (mek@med.md). Acromioclavucular joint separation. N Engl J Med. 2017;376:e1

A, B: Schwellung über der linken Schulter. C: Sprengung des Akromioklavikulargelenks im Röntgenbild. 